\title{
Antithrombotic therapy after TAVI
}

The combination of oral anticoagulation and the antiplatelet drug clopidogrel increases the risk of bleeding after transcatheter aortic valve implantation (TAVI) compared with oral anticoagulation alone, according to the results of the POPular TAVI trial cohort B, which were presented at the ACC Scientific Sessions 2020.

Patients undergoing TAVI often have atrial fibrillation and, for those with an indication, current clinical guidelines recommend oral anticoagulation with or without aspirin or clopidogrel. The addition of an antiplatelet agent is intended to reduce the risk of thrombotic events but might be outweighed by an increase in bleeding.

POPular TAVI cohort B included patients undergoing TAVI with a preexisting indication for anticoagulation (cohort A includes those without an indication for anticoagulation and has not yet been completed). Patients receiving oral anticoagulation were randomly assigned to 3 months with or without clopidogrel therapy after TAVI and were followed up for 12 months. Bleeding occurred in $21.7 \%$ of the 157 patients receiving oral anticoagulation therapy alone compared with $34.6 \%$ of the 156 patients receiving oral anticoagulation plus clopidogrel therapy (risk ratio (RR) 0.63, 95\% CI 0.43-0.90, $P=0.01)$. Non-procedure-related bleeding (which included bleeding at the puncture site) occurred in $21.7 \%$ and $34.0 \%$ of patients in each group, respectively (RR 0.64, 95\% CI $0.44-0.92, P=0.02)$. A secondary end point of myocardial infarction, ischaemic stroke or death from cardiovascular causes occurred in $13.4 \%$ and $17.3 \%$ of patients in each group, respectively, which met the prespecified criteria for non-inferiority of no clopidogrel therapy.

"The ideal would be an anticoagulant regimen that minimizes the risk of death, stroke, myocardial infarction or permanent disability, whether resulting from thrombosis or bleeding," comments Frederick Fiet in an accompanying editorial in NEJM. "These are the events that matter most to patients."

Gregory B. Lim

ORIGINAL ARTICLE Nijenhuis, V. J. et al. Anticoagulation with or without clopidogrel after transcatheter aortic-valve implantation. N. Engl.J.Med. https://doi.org/10.1056/NEJMoa 1915152 (2020)

\section{CARDIAC REGENERATION}

\section{An acellular artificial cardiac patch for myocardial repair}

A limitation of cell-based therapy for myocardial repair is the low retention and engraftment of transplanted cells at the target site. Cardiac patches, which consist of cells embedded in a scaffold material, applied to the surface of the heart can overcome this problem, but cell viability limits long-term storage and mass production. Now, Ke Cheng and colleagues report the creation of an 'off-the-shelf', cell-free, artificial cardiac patch that is easy to store and improves outcomes in rat and pig models of myocardial infarction (MI).

To create the artificial cardiac patch, the researchers first produced a myocardial extracellular matrix scaffold from decellularized porcine myocardium, which retains its native ultrastructural and material properties. Next, they produced synthetic cardiac stromal cells (CSCs) by encapsulating human CSC-secreted factors in biodegradable microparticles. These synthetic CSCs were then embedded in the extracellular matrix scaffold. Being fully acellular, the cardiac patch could be cryostored for at least 28 days without detrimental effects on the mechanical properties or therapeutic efficacy.

Transplantation of the artificial cardiac patch into a rat model of acute $\mathrm{MI}$ reduced the amount of scarring, promoted angiomyogenesis and increased left ventricular ejection fraction compared with animals that received a cardiac patch with no synthetic $\mathrm{CSC}$ s or those that received no treatment. Moreover, the artificial cardiac patch was biocompatible and safe in a pig model of acute $\mathrm{Ml}$ and resulted in a smaller infarct size, less myocardial fibrosis and a larger improvement in ejection fraction than in pigs that received no treatment. The researchers conclude that the artificial patch provides "a clinically feasible, easy-to-store, and cell-free alternative to myocardial repair using cell-based cardiac patches".

Gregory B. Lim

ORIGINAL ARTICLE Huang, K. et al. An off-the-shelf artificial cardiac patch improves cardiac repair after myocardial infarction in rats and pigs. Sci. Transl Med. 12, eaat9683 (2020)

\section{HYPERTENSION}

\section{BP-lowering with renal denervation}

In patients with uncontrolled hypertension who are not receiving antihypertensive drugs, catheter-based renal denervation significantly lowers blood pressure (BP) levels compared with a sham procedure. This finding from the SPYRAL HTN-OFF MED Pivotal trial was presented at the virtual ACC 2020 Scientific Sessions.

Investigators of the international, randomized, sham-controlled, proof-ofconcept SPYRAL HTN-ON MED trial had previously showed that renal denervation in the main renal arteries significantly reduced $\mathrm{BP}$ compared with a sham procedure. The subsequent SPYRAL Pivotal trial was designed to evaluate the efficacy of renal denervation in patients who were not receiving antihypertensive medication. Patients with office systolic BP (SBP) of $150-180 \mathrm{mmHg}$ were randomly assigned to renal denervation $(n=166)$ or a sham procedure $(n=165)$. The primary and secondary efficacy end points were changes in 24-h SBP and office SBP, respectively, from baseline to 3 months after the procedure.

At 3 months, both end points were met with a posterior probability of superiority $>0.999$. The treatment difference between the two groups was $-3.9 \mathrm{mmHg}$ (Bayesian 95\% credible interval $-6.2 \mathrm{mmHg}$ to $-1.6 \mathrm{mmHg}$ ) for 24-h SBP and $-6.5 \mathrm{mmHg}(-9.6 \mathrm{mmHg}$ to $-3.5 \mathrm{mmHg})$ for office SBP, both favouring the renal denervation group. Importantly, treatment differences between groups for 24-h SBP were consistent among all the baseline characteristic subgroups assessed. No major device-related or procedure-related adverse events were observed in either treatment group.

"The SPYRAL Pivotal trial is, to the best of our knowledge, the largest randomized trial to show the superiority of catheter-based renal denervation, compared with a sham procedure, to lower blood pressure in the absence of antihypertensive medications," comment the investigators. "Long-term efficacy and safety will continue to be followed up for 3 years."

Karina Huynh

ORIGINAL ARTICLE Böhm, M. et al. Efficacy of catheterbased renal denervation in the absence of antihypertensive medications (SPYRAL HTN-OFF MED Pivotal): a multicentre, randomised, sham-controlled trial. Lancet https://doi.org/ 10.1016/S0140-6736(20)30554-7 (2020) 